\section{Auf dem Weg zur institutionalisier- ten Nicht-Institutionalisierung?}

\author{
Was in den 1970er Jahren als Konfrontationskurs der Neven Sozialen Bewe- \\ gungen gegen das politische System begonnen hatte, ist heute ein nicht mehr \\ wegzudenkender Bestandteil des politischen Alltags in Deutschland geworden. \\ Nichtregierungsorganisationen machen Politik. Auch Politiker versuchen inzwi- \\ schen, sich dies als politische Ressource nutzbar zu machen. Dies wird von neu- \\ en Governance-Konzepten offensiv aufgegriffen und verändert die Herausfor- \\ derungen für politische Steuerung.
}

$\mathrm{N}$

Von Carolin Drube

vernmental organisations, NGOs) sind vor dem Hintergrund einer langen Entstehungsgeschichte keine Erscheinung des globalisierten Zeitalters. Dennoch wird die Diskussion um den politischen Einfluss und die Legitimität von nichtstaatlichen Organisationen so heftig wie nie zuvor gefuihrt. Während sich für manche in dieser $\mathrm{Zu}$ sammenarbeit eine Form der Zivilgesellschaft wider spiegelt, kritisieren andere deren nicht demokratisch legitimierte Einflussnahme. Der Kontroverse liegt eine neue Qualität der Kooperationsbeziehungen zwischen Staat und Gesellschaft zugrunde, die das Resultat eines veränderten Selbstverständnisses der Umweltschutzorganisationen und alternativer Formen der politischen Steuerung ist. Diese Wechselwirkung hat die Einbeziehung von nichtstaatlichen Akteuren zu einer politischen Notwendigkeit werden lassen (1).

\section{Vom Protestakteur zum Kooperationspartner}

Spätestens seit dem Gipfel von Rio 1992 rücken Umweltorganisationen verstärkt ins öffentliche Bewusstsein und werden zu politischen Partnern der Entscheidungsträger. Einfluss nehmen Umweltorganisationen seitdem in erster Linie durch Wissen und die Sensibilisierung einer kritischen Öffentlichkeit. Wurden Aufsehen erregende Aktionen vor 20 Jahren noch als Affront gegen die Politik verstanden, so fällt dies heute unter die Kategorie des politischen Monitorings. Vor allem vor dem Hintergrund der Verlagerung von politischen Entscheidungen auf die europäische und internationale Ebene wird Umweltorganisationen mit dieser Aufgabe eine wichtige demokratische
Funktion zugesprochen (2). Indem sie für mehr Transparenz in staatlichen Entscheidungsprozessen sorgen, verhindern sie, dass politische Beschlüsse hinter dem Rücken der Öffentlichkeit gefällt werden. Dabei steht ihnen eine Bandbreite an Instrumenten der Öffentlichkeitsarbeit wie die Verbreitung von Informationsbroschüren, Weiterbildungsangebote und öffentlichkeitswirksame Aktionen zu Verfügung.

\section{- Entscheidend ist der Sachverstand}

Während traditionelle korporatistische Akteure wie Industrieverbände und Gewerkschaften bereits seit Jahrzehnten in politische Entscheidungsprozesse eingebunden sind und über ausreichend finanzielle Mittel und Sanktionsmechanismen verfügen, entwickelt sich erst allmählich ein Netzwerk zwischen den politischen Akteuren und den Umwelt-NGOs. Die NGO-Bewegung hat aber auch deutlich werden lassen, dass politische Einflussnahme außerhalb der Exekutive möglich ist. Sachkompetenz ist heute zum zentralen Merkmal ihres politischen Einflusspotenzials geworden. Ohne spezielles Wissen und einen Vorsprung an Informationen sind umweltpolitische Nichtregierungsorganisationen weitgehend machtlos. Bereits im Vorfeld von Konferenzen unterbreiten Umweltorganisationen Vorschläge für Regierungserklärungen, nehmen Kontakt zu Delegierten auf und versuchen so, die politische Agenda zu beeinflussen. Die Bereitstellung entscheidungsrelevanter Expertise spielt eine entscheidende Rolle bei der Vorbereitung von Beschlüssen und Gesetzen, ebenso wie beim Setzen von Begriffen und bei der Strukturierung von Themen. Wie erfolgreich ihre Lobbyarbeit letztendlich ist, zeigt sich, wenn sich Sachzusammenhänge, Empfehlungen und Begriffe in politischen Abkommen und Konventionen wieder finden.

\section{Die Governance-Debatte}

Die ausdifferenzierte Wahrnehmung politischer Steuerungsmöglichkeiten und die Neudefinition des Verhältnisses zwischen Staat und Gesellschaft hat dazu geführt, dass heute NGOs als politischer Akteur angesehen werden. Die veränderten Kooperationsbedingungen sind sowohl Ausdruck als auch Impuls der Diskussion um neue Formen der politischen Steuerung. Politische Steuerung wird in der Governance-Debatte als Bündelung der gesellschaftlichen, wirtschaftlichen und politischen Akteure zur gemeinsamen Problemlösung jenseits territorialer Grenzen verstanden. Das Konzept beruht auf konsensbasierten Regelungen und diskursiven Aushandlungsprozessen und läßt traditionelle Formen der command-and-control Regulation in den Hintergrund treten (3). Die Aktualität der Governance-Debatte ist eine Reaktion auf die Krise staatlicher Leistungsfähigkeit, die sich in unsicheren Ursache-Wirkungs-Zusammenhängen, eine Vielzahl von Handlungsalternativen sowie in einer sinkenden Geltungsreichweite politischer Entscheidungen niederschlägt (4).

\section{Wechselseitige Annäherung}

Dieser Steuerungsansatz hat zu einer Öffnung der Politik beigetragen und dazu geführt, dass der politische Beteiligungsanspruch der Nichtregierungsorganisationen heute keine kommunikative Einbahnstraße mehr ist. Nicht nur Umweltorganisationen suchen den Kontakt zu den politischen Entscheidungsträgern sondern auch umgekehrt. Einhergehend mit der zunehmenden Komplexität von Sachfragen können politische Akteure nur noch bedingt Antworten auf anfallende Fragen und Problemkontexte finden, weshalb sie mehr und mehr auf Expertenwissen angewiesen sind. Darüber hinaus versprechen sich die politischen Entscheidungsträger von der Einbeziehung nichtstaatlicher Akteure eine Aufbesserung ihres Images. Während Meinungsumfragen gesellschaftlichen Vertrauensschwund in die politische Problemlösungsfähigkeit diagnostizieren, erfreuen sich Umweltorganisationen nach wie vor großer Beliebtheit und Integrität bei der deutschen Bevölkerung (5). Dem Wähler soll die neue Kooperation frischen Wind bei der Problemlösung, die Auflösung verkrusteter Verwaltungsstrukturen und Effizienzgewinne signalisieren. Die Hinwendung zum Dialog mit Nichtregierungsorganisationen ist ein parteiü- 
bergreifendes Phänomen, wenngleich sich deutliche Unterschiede hinsichtlich der Wahl der Gesprächspartner verzeichnen lassen.

Umweltorganisationen haben auf die veränderten politischen Rahmenbedingungen und die daraus resultierenden Gelegenheiten mit einer Neubewertung ihres institutionellen Selbstverständnisses reagiert. Durch eine gezielte Strategie, die stärker auf Kooperation denn auf Konfrontation ausgerichtet ist, haben Umwelt-NGOs ihre politischen Zugangsmöglichkeiten sukzessiv genutzt und ausgeweitet (6). Doch trotz all dieser Tendenzen beginnt erst langsam ein Nachdenken über die strukturierte und formalisierte Einbeziehung gesellschaftlicher Akteure.

\section{- Erste Ansätze einer Institutionalisierung}

In ihrer Koalitionsvereinbarung verpflichtete sich die rot-grüne Regierung 1998 zu einem strukturierten Dialog mit umweltpolitischen Akteuren. Bis heute ist dieser jedoch nur ansatzweise - zum Beispiel im Rat für Nachhaltige Entwicklung verwirklicht. Die 17 Ratsmitglieder, von denen drei dem Kreis der Umwelt-NGOs zuzuordnen sind, haben die Aufgabe, die Bundesregierung zu beraten und zur Konkretisierung der Nachhaltigkeitspolitik beizutragen, um das Konzept einer breiten Öffentlichkeit zugänglich zu machen. Damit ist zweifelsohne der Wunsch verbunden, die Politik der Nachhaltigkeit jenseits von parteipolitischen Querelen voranzutreiben und Reformvorschläge in Abstimmung mit gesellschaftlichen Akteuren abzusichern. Zwar lässt sich tendenziell mit dem Regierungswechsel eine Öffnung des politischen Entscheidungsprozesses erkennen. Jedoch darf dies nicht darüber hinwegtäuschen, dass sich die langsam beginnende Institutionalisierung von Zugangsmöglichkeiten auf eine kleine, ausgesuchte Gruppe von nichtstaatlichen Akteuren beschränkt.

\section{Grenzen der Macht}

Wie groß ist folglich der politische Einfluss von Umweltorganisationen? Die Euphorie nach dem großen Verhandlungserfolg von Rio ist abgeklungen. Die Hoffnung der Nichtregierungsorganisationen auf einen breiten, institutionalisierten und formalisierten Dialog nach dem Regierungswechsel hat sich nicht bestätigt. Doch auch wenn ein qualitativer Fortschritt in den Beziehungen zwischen Staat und Gesellschaft nach dem Regierungswechsel nur für einige wenige zutrifft, zeigt die gestiegene Anzahl und Kontinuität der Treffen, dass die Kooperation zwischen staatlichen und nichtstaatlichen Akteuren keine ,politische Eintagsfliege" mehr ist. Mittlerweile nehmen Umweltorganisationen häufig als umweltpolitische Berater weitab von der medialen Aufmerksamkeit an politischen Entscheidungsprozessen teil.

So lange es keine formalisierten oder zumindest institutionalisierten Zugangsmöglichkeiten für Nichtregierungsorganisationen gibt, wird ihr politischer Einfluss weiterhin situativ bedingt, das heißt von der politischen Krisenlage abhängig sein, ebenso wie von der Brisanz des Themas und der Bereitschaft der politischen Entscheidungsträger, NGOs einzubinden. Gerade vor dem Hintergrund der Terroranschläge in den USA ist deutlich geworden, dass sich politische Prioritäten von heute auf morgen ändern können und dass unterschiedliche Situationen auch unterschiedliche Steuerungsmechanismen bedingen. Dabei sind diskursive Aushandlungsprozesse eben nur eine von vielen möglichen Steuerungsalternativen für politische Akteure.

\section{- Ausblick: „Jede Regierung ist nur so gut wie ihre..." NGO?}

Insgesamt sind Umweltorganisationen zu einem relevanten politischen Akteur geworden. Freilich ist noch weitgehend offen, wie eine institutionalisierte Verzahnung von gesellschaftlicher und politischer Ebene aussehen kann. Die Diskussion um Global Governance und die Zusammenarbeit mit nichtstaatlichen Organisationen hat die Politik um eine neue, unkonventionelle Steuerungsform bereichert. Diskursive Aushandlungsprozesse greifen vor allem dort, wo es beiden Seiten um die Suche nach der besten Lösung und nicht um parteipolitische Interessenkonflikte geht. Die Aufgabe für die Zukunft wird die Suche nach Formen ,institutionalisierter Nicht-Institutionalisierbarkeit“ (7) sein, um die Vorteile von Diskursen unter externer Beteiligung strategisch nutzen zu können.

Die Schwierigkeiten liegen auf der Hand. Bei einer formalisierten Beteiligung laufen Umweltorganisationen Gefahr, die eigentliche Begründung ihres politischen Einflusses zu unterlaufen. Gelten sie heute noch als politisch unabhängig und dem Thema verpflichtet, kann der politische Bedeutungszuwachs morgen ihre eigene Unterwanderung bedeuten. NGOs müssen daher unabhängig vom Staat und seinen finanziellen Mitteln sein. Dies ist auch im Sinn der Politik, da die Qualität von NGOs in hohem Maße an deren Eigenständigkeit gekoppelt ist. Nur so lässt sich auch gewähr- leisten, dass umweltpolitische Interessengruppen weiterhin nach alternativen und unkonventionellen Lösungsansätzen suchen.

Die staatlichen Akteure müssen dabei die Bereitschaft mitbringen, neue Wege in der Problemlösung zu gehen. Die Kooperationsebenen könnten je nach Thema relativ lose Gesprächszirkel, entscheidungsvorbereitende Netzwerke und gemeinsame Projekte mit dem Ziel, einen kontinuierlichen und transparenten Austausch zwischen Staat und Gesellschaft zu gewährleisten, sein.

Auf dieser Basis könnte die Politik an gesellschaftlicher Akzeptanz zurückgewinnen, was auch dazu führen könnte, dass Aufgabendelegation an und Einbeziehung von nichtstaatlichen Akteuren keinen Verlust staatlicher Handlungsmacht, sondern durchaus einen Gewinn an Steuerungsfähigkeit bedeutet.

\section{Anmerkungen}

(1) Vgl. hierzu ausführlich Drube, C.: Die Zugangsmöglichkeiten von Umweltorganisationen zum politischen Entscheidungsprozess: Das Beispiel der Bundesrepublik Deutschland. Augsburg 2000 (unveröffentlichte Diplomarbeit).

(2) Vgl. dazu auch Altvater, E./ Brunnengräber, A./ Walk, H.: Substitut des Staatsvolks? In: Ökologisches Wirtschaften, Nr. 2/1998, S. 23-25.

(3) Der Begriff Global Governance wurde im Wesentlichen von der Commission on Global Governance geprägt. Vgl.

Stiftung Entwicklung und Frieden (Hrsg.): Nachbarn in Einer Welt. Der Bericht der Kommission für Weltordnungspolitik, Bonn 1995.

(4) Vgl. dazu auch den Schwerpunkt "Governancestrukturen für Nachhaltigkeit" der Ausgabe 3-4/2001 von Ökologisches Wirtschaften.

(5) Vgl. z.B. Bundesministerium für Umwelt, Naturschutz und Reaktorsicherheit (Hrsg.): Umweltbewusstsein in Deutschland 1998. Ergebnisse einer repräsentativen Umfrage, Bonn 1998.

(6) Vgl. dazu Drube a.a.0. sowie aktuell die Ausgabe 72 der Politischen Ökologie "Vom David zum Goliath? NGOs im Wandel".

(7) Sarcinelli, Ulrich: Repräsentation oder Diskurs? Zur Legitimität und Legitimitätswandel durch politische Kommunikation, in: Zeitschrift für Politikwissenschaft, 8. Jg. (1998), Heft 2, S. 547-567.

\section{Die Autorin}

Carolin Drube studierte Politikwissenschaften und ist seit 2000 Mitarbeiterin am Institut für Management und Umwelt in Augsburg.

Kontakt: imu, Gratzmüllerstr. 3, 86150 Augsburg, Tel. 0821/ 34366-16, Fax -39,

E-Mail: drube@imu-augsburg.de 
(c) 20I0 Authors; licensee IÖW and oekom verlag. This is an article distributed under the terms of the Creative Commons Attribution Non-Commercial No Derivates License (http://creativecommons.org/licenses/by-nc-nd/3.o/), which permits unrestricted use, distribution, and reproduction in any medium, provided the original work is properly cited. 